\title{
Autoridad política y cambio de política pública: política de agua y saneamiento en Ecuador desde el enfoque de redes de política
}

\author{
Political authority and policy change: Water and sanitation policy \\ in Ecuador from the policy networks approach
}

\author{
W. SANTIAGO ALBUJA-ECHEVERRÍA \\ FLACSO Ecuador
}

MARÍA BELÉN ALBORNOZ

FLACSO Ecuador

\section{Cómo citar/Citation}

Albuja-Echeverría, W. S. y Albornoz, M. B. (2020). Autoridad política y cambio de política pública: un análisis desde el enfoque de redes de política. Revista Española de Ciencia Política, 52, 147-175. Doi: Disponible en: https://doi.org/10.21308/recp.52.06

\section{Resumen}

Dentro del campo de estudio de las políticas públicas ha surgido en los últimos años un creciente interés por analizar por qué y cómo cambian estas. El objetivo de este artículo es estudiar el cambio de la política pública de agua y saneamiento en Ecuador entre los ańos 2008 y 2015, con la finalidad de explicar las principales causas de su transformación y sus consecuencias. Para ello, utilizamos el modelo ideacional (Kisby, 2007) de redes de política en sus tres niveles: contexto, estructura y resultados de la política. La hipótesis que sostenemos aquí es que los instrumentos de la política se transforman debido a la intervención externa del presidente de la república, y que dicha intervención reorganiza el contexto de la política, la estructura de la red y los recursos dentro del proceso de toma de decisiones. El cambio de la política basado en la intervención directa de una autoridad política puede entonces anular los consensos de las redes de política, transformar los procesos de toma de decisiones, reformular los instrumentos y reemplazar las creencias programáticas de los actores (Marsh y Smith, 2000).

Palabras clave: cambio de política, autoridad política, redes de política pública, agua y saneamiento. 


\begin{abstract}
In the field of public policies studies, there has lately emerged a growing interest in analyzing why and how public policy change. The aim of this article is to study the change of water and sanitation public policy in Ecuador between 2008 and 2015, in order to explain the main causes of its transformation and its consequences. To this aim, the ideational model (Kisby, 2007) of policy networks is used at three levels: context, network and policy results. The hypothesis held here is that policy instruments change due to the external intervention of the President of the Republic which, in turn, reorganizes the policy context, network structure and resources of the decision-making process. Policy change based on the direct intervention of a political authority can thus cancel the consensus within the policy networks, transform the decision-making process, change the policy instruments and replace the actors' programmatic beliefs (Marsh and Smith, 2000).
\end{abstract}

Keywords: policy change, political authority, policy networks, water and sanitation.

\title{
INTRODUCCIÓN
}

De manera amplia, las políticas públicas hacen referencia a las estrategias o acciones ejecutadas por el Estado para la solución de un problema determinado, a través de la combinación de una gama de incentivos y formas de gestión pública (Méndez, 2010: 121). En los últimos años, en el campo del análisis de las políticas públicas ha surgido un gran interés por estudiar el cambio de política pública. Dentro de esta tendencia, este artículo analiza el cambio de la política de la cobertura de agua y saneamiento en Ecuador, con la finalidad de explicar las principales causas de su transformación y las principales consecuencias. La literatura que se ocupa del cambio de la política pública presenta varios enfoques de análisis que no son excluyentes entre sí, pero cuya identificación ayuda a definir la alineación metodológica que utilizar en cada caso. Entre los más importantes tenemos el enfoque racionalista, el cognitivista y el neoinstitucionalista (Fontanine, 2015). Este trabajo se desarrolla bajo el paraguas del neoinstitucionalismo; en particular, en la propuesta de los tres tipos de cambio de Hall (1993) y en el análisis de redes de política (Knoke, 1993; Klijn, 1998; Bressers y O’Toole, 1998; George y Bennett, 2004; Montpetit, 2005; Kisby, 2007).

Si bien el análisis de redes de política no es nuevo dentro del neoinstitucionalismo, otros enfoques han sido más utilizados para mostrar cómo se instrumenta el cambio de las políticas, tales como la teoría de las corrientes múltiples (Kingdon, 2003; Capano, 2009), la teoría de los equilibrios puntuales (Baumgartner y Jones, 2002) o la teoría de las coaliciones promotoras (Sabatier, 1999; Ordoñez-Matamoros, 2013). Nuestra opción teórica se fundamenta en que la acción de los actores no se debe a una ventana de oportunidad específica ni al desarrollo de una agenda impulsada por los distintos sectores de la política. Tampoco se puede explicar el cambio a través de la combinación de las variaciones en la percepción pública debido a una crisis externa. Y finalmente, aunque tomamos en cuenta las ideas programáticas de los actores, no son 
sus sistemas de creencias ni sus valores los que definen el cambio de la política en el caso de estudio que presentamos en este trabajo. Por tanto, los enfoques antes mencionados no son útiles para explicar el problema que planteamos en este texto.

En cambio, la teoría de redes de política se concentra en elucidar las relaciones de influencia y la dirección de las interacciones que ocurren en los procesos de toma de decisiones (Lieberman, 2000; Klijn y Koppenjan, 2016; Eberhad et al. 2017; Howlett et al., 2017) y, sobre todo, nos ofrece las nociones de impacto exógeno (Real Dato, 2009) y de autoridad política (Marsh y Smith, 2000; Richardson, 2000) que nos resultan fundamentales para mostrar la causalidad entre la variable independiente $y$ dependiente. El análisis de redes asume que los actores participan en sistemas sociales, conectándose con otros actores cuyas relaciones tienen una importante influencia en el comportamiento de cada uno (Knoke y Yang, 2008). Igualmente, las redes afectan las acciones, percepciones y creencias de los actores por medio de mecanismos estructurales que son construidos socialmente, ofreciendo la posibilidad de explicar tanto las estructuras como los cambios que ocurren a nivel micro con las alteraciones estructurales a nivel macro (Marsh y Rhodes, 1992).

En términos metodológicos, este estudio de caso propone como variable dependiente el cambio de la política pública, y como variable independiente la intervención de la autoridad politica en el cambio de los instrumentos de la política. Se recurre a la aplicación de dos criterios del caso típico de Gerring (2008): a) la búsqueda de un caso representativo que permita analizar la transformación de la política a partir de la alteración de sus instrumentos, y b) contar con una hipótesis probable. Considerando lo expuesto, se escoge como caso de estudio la política de ampliación de la cobertura de agua y saneamiento en el Ecuador entre 2008 y 2015. Elegimos este periodo ya que en el año 2008 se aprueba la nueva Constitución, que establece la garantía del derecho al agua y saneamiento. Ello fortalece la implementación del programa Prosaneamiento, principal instrumento crediticio dirigido hacia los Gobiernos Autónomos Descentralizados (GAD) municipales con la finalidad de ampliar la cobertura de estos servicios básicos. En el año 2014, Prosaneamiento es alterado por la eliminación del componente crediticio no reembolsable, que incentivaba a los GAD para acceder a estos créditos bajo condiciones preferenciales. A raíz de ello, en el año 2015 se construye la Estrategia Nacional de Agua y Saneamiento, que establece orientaciones para las intervenciones en este sector. Se propone como hipótesis que el cambio de primer orden de la política se debe a la intervención de una autoridad política externa a las redes de política en la implementación de la política pública.

Este trabajo está estructurado en cuatro secciones. La primera sección introduce las características de la política pública de agua y saneamiento en Ecuador. La segunda sección aborda el modelo de análisis que contempla las opciones teóricas y metodológicas

1. En términos de Marsh y Smith: «La autoridad política, probablemente, es la más importante restricción; siendo difícil, aunque no imposible, ignorar la presión política directa para el cambio» (2000: 8). 
elegidas a fin de desarrollar esta investigación. La tercera sección consiste en la aplicación del modelo de análisis en tres niveles: a) contexto: expone los hechos que han configurado la política pública de agua y saneamiento; b) estructura y agencia: analiza los procesos de toma de decisiones y selección de instrumentos en función del contexto, ideas programáticas, recursos y habilidades de los actores, y c) red y resultado: explica las causas y consecuencias del cambio de política pública. Por último, la cuarta sección presenta las conclusiones y hallazgos.

\section{LA POLÍTICA DE COBERTURA DE AGUA Y SANEAMIENTO EN EL ECUADOR}

El acceso a agua y saneamiento constituye un derecho que posibilita el cumplimiento de otros derechos tales como la salud, la alimentación, el hábitat y vivienda digna, la educación, y el empleo, entre otros. En Ecuador, el $38 \%$ de la pobreza y el $64 \%$ de la pobreza extrema por necesidades básicas insatisfechas se explica por la dimensión correspondiente a las carencias de agua y saneamiento (SENPLADES, 2014a: 6). La cobertura de estos servicios básicos a nivel nacional oculta grandes brechas territoriales entre las áreas urbana y rural.

Los orígenes de la política de agua y saneamiento se remontan a la década de los ańos cuarenta del pasado siglo, con la llegada del Servicio Cooperativo de los Estados Unidos a algunos países de la región latinoamericana —entre ellos Ecuador- para la construcción de la primera infraestructura hidrosanitaria en el país. El Instituto Ecuatoriano de Obras Sanitarias (IEOS) se creó en el año 1965, con las atribuciones de planificación, diseño y construcción de obras para la ampliación y mantenimiento de estos servicios. Históricamente la competencia de la prestación de agua y saneamiento ha estado a cargo de los GAD, cuyo mecanismo de financiación fue el Banco del Estado (BDE) desde 1979. Con la eliminación del IEOS en 1992, el Ministerio de Desarrollo Urbano y Vivienda (MIDUVI) adoptó la rectoría de este sector. En el año 2004 se formula la Política Nacional de Agua Potable y Saneamiento como política de Estado, con un espectro de acción que abarca desde el establecimiento de una guía tarifaria hasta la ejecución de programas con recursos propios y provenientes de la cooperación. Su implementación tiene bajos resultados debido a la escasa participación de los actores involucrados y a la ingobernabilidad que vivió el país en el período 2000-2006. Ecuador llega a tener cuatro presidentes en siete años, lo que repercute en una planificación y administración inestables.

Una vez que Rafael Correa llegó a la presidencia de la República en el 2006, se presentó el proyecto para el reemplazo de la Constitución de 1998, y en 2008 el país adoptó una nueva Constitución. La nueva norma constitucional establece un Estado de derechos que promueve y garantiza los derechos individuales, colectivos y de la naturaleza; lo que marca el inicio del período de análisis de la política escogida debido al sitial que se le otorga al derecho al agua y saneamiento. El agua adquiere el estatus de patrimonio nacional estratégico de uso público y de derecho de las personas, que 
debe ser velado por el Estado mediante una política nacional de agua y saneamiento. En este contexto, se ratifica a los GAD la competencia de la prestación de estos servicios, y se le otorga a la Secretaría Nacional del Agua (SENAGUA) la rectoría y la formulación de la política nacional en lugar del MIDUVI. Con el objeto de mejorar la cobertura especialmente en el área rural, en 2007 se creó el programa Prosaneamiento, con la financiación del BDE para preinversión, inversión y fortalecimiento de capacidades. Esta financiación tiene un importante componente no reembolsable y cuenta con niveles de subvención a los GAD, para incentivar la construcción y mantenimiento de obras vinculadas a la prestación del servicio de agua y saneamiento.

TABLA 1.

COBERTURA DE AGUA Y SANEAMIENTO 2007 Y 2014

\begin{tabular}{llll}
\hline Indicador & Desagregación & $\mathbf{2 0 0 7}$ & $\mathbf{2 0 1 4}$ \\
\hline \multirow{3}{*}{ Viviendas con agua por red pública } & Nacional & $71,7 \%$ & $77,2 \%$ \\
\cline { 2 - 4 } & Urbano & $90,4 \%$ & $93,4 \%$ \\
\cline { 2 - 4 } & Rural & $31,1 \%$ & $40,9 \%$ \\
\hline \multirow{3}{*}{ Viviendas con alcantarillado sanitario } & Nacional & $55,2 \%$ & $61,4 \%$ \\
\cline { 2 - 4 } & Urbano & $73,8 \%$ & $77,5 \%$ \\
\cline { 2 - 4 } & Rural & $14,7 \%$ & $25,5 \%$ \\
\hline
\end{tabular}

Fuente: INEC. Encuesta Nacional de Empleo, Desempleo y Subempleo (2007, 2014).

A pesar de los buenos resultados en cuanto al incremento de coberturas (tabla 1), en el 2014 el presidente decidió promover la eliminación de los subsidios en la financiación de la política, lo que ocasiona un cambio en la selección de los instrumentos, fenómeno que motiva esta investigación. Este artículo explica la incidencia de la autoridad del presidente en la toma de decisiones en tres momentos: a) el contexto de la política, sus actores, redes e instrumentos; b) las relaciones de influencia y dominación en la toma de decisiones desde las agendas de las redes de política y la intervención del presidente; y c) el cambio de política dado por la alteración de sus instrumentos y la reorganización de sus recursos.

\section{MODELO DE ANÁLISIS}

Para identificar si nos encontramos frente a un cambio incremental o radical, utilizamos la tipología de Hall (1993) de cambio de primer, segundo y tercer orden. El cambio de primer orden ocurre cuando los instrumentos son ajustados en función de la experiencia pasada y de nuevos conocimientos que responden a decisiones técnicas, manteniendo los instrumentos y los objetivos de la política intactos. El cambio de segundo orden hace referencia a la creación de nuevos instrumentos mientras que los objetivos generales de la política siguen siendo los mismos (ibid:: 278). El cambio de 
tercer orden, también denominado paradigmático o revolucionario, implica cambios simultáneos en los tres componentes de la política; por tanto, es el resultado de un cambio de paradigma como consecuencia de la insatisfacción de experiencias pasadas, de la influencia de nuevas ideas y de la presencia de crisis institucionales. Este cambio está vinculado a eventos exógenos (ibid:: 279). El caso que presentamos en este artículo se refiere a un cambio de primer orden. El modelo de análisis escogido para explicar por qué ocurre este cambio de la política se basa en el modelo de redes de política (Klijn, 1998; Kisby, 2007; Bressers y O’Toole, 2007) y en la noción de impacto exógeno (Real Dato, 2009) y de autoridad política (Knoke, 1993; Marsh y Smith, 2000; Richardson, 2000). Sostenemos que una autoridad política ajena a las redes de política encargadas de la implementación de una política sectorial concreta logra imponerse en la toma de decisiones y transforma la implementación a través de un giro en la selección de los instrumentos.

Esta investigación se centra en el estudio del cambio de política a partir de la alteración de sus instrumentos, entendidos como «técnicas de gobernanza que, de una u otra forma, incluyen la utilización de la autoridad del Estado o su limitación consiente» (Howlett et al., 2006: 130). Si bien los instrumentos tienen un alto contenido técnico, es importante señalar que también están altamente influenciados por el deseo de los actores de la política de mantener o alcanzar el poder político. Según Bressers y O’Toole (2007), existe un vínculo entre las relaciones de poder del contexto sociopolítico y la toma de decisiones sobre la selección de los instrumentos de la política, puesto que «los instrumentos están usualmente destinados a alterar el status quo, presentando implicaciones con respecto a cambios en la distribución de costos y beneficios vía gobernanza» (ibid.: 133). La única forma en que se alteren las relaciones de poder de los actores que configuran las redes de política es que factores exógenos causen directamente el cambio de la política, lo que Real Dato denomina el mecanismo de impacto exógeno, donde el cambio se produce "como consecuencia de acontecimientos originados más allá de los límites del subsistema, en otros ámbitos de acción con los que se relaciona» (2009: 135). Sin embargo, si el núcleo de decisiones de las redes se omite, las decisiones externas pueden moldear directamente los elementos del cambio, ocasionando que la toma de decisiones suba a un nivel superior o a una arena de acción exterior.

Marsh y Smith (2000) sostienen que existen factores contextuales económicos, ideológicos y políticos que pueden influir sobre el proceso de la política. Inspirados en la propuesta de Richardson (2000) sobre la importancia del poder del Estado, las transformaciones en el comportamiento de los grupos de interés y el rol del conocimiento y de las ideas, estos autores proponen que la autoridad política probablemente es la restricción más importante del contexto de la toma de decisión de las redes de política, por lo que no es posible ignorar la presión política directa en el cambio de la política (Marsh y Smith, 2000: 8). A partir de estas nociones teóricas, para efectos de esta investigación, la autoridad política actúa como un actor particular que es vocero de sí mismo y no forma parte del subsistema en donde se discute la política pública, pero es capaz de tomar decisiones e imponerse jerárquicamente 
desde un ámbito de acción superior. Para analizar la relación entre las variables dependiente e independiente. se ha escogido el enfoque de redes de política debido a su fortaleza teórico-metodológica para explicar cómo el contexto, los actores y sus relaciones inciden en un determinado resultado de política: en este caso, el cambio de primer orden de la política. Las redes de política permiten mostrar cómo el resultado de la política afecta al contexto, a los actores y sus relaciones, reorganizando el proceso de la política pública.

Definimos a las redes de política como estructuras sociales que mantienen patrones de relaciones más o menos estables entre actores independientes en torno a problemas o programas políticos (Knoke, 1993; Klijn, 1998). Las redes nos muestran la estructura de las relaciones que establecen los actores y las instituciones durante el cambio de la política pública, y esta estructura es fundamental para comprender los intereses de los actores, sus preferencias y las negociaciones de influencia y dominación que ejercen entre ellos (Knoke, 1993). Las redes son portadoras de agencias y mantienen una visión del mundo que funciona como una limitación estructural derivada de una cultura común que condiciona la acción de los miembros de la red. Por ello, es fundamental entender cómo se producen, reproducen y cambian los patrones de comportamiento dentro de la red; por qué las preferencias de los actores y sus construcciones discursivas ayudan a generar los límites y las oportunidades que la estructura de la red ofrece, y cómo dicha estructura condiciona las preferencias y los discursos de los actores (Marsh y Smith, 2000).

Utilizamos el modelo ideacional de redes de política de Kisby (gráfico 1) para mostrar cómo se estructuran las redes de política de ampliación de la cobertura de agua y saneamiento en el Ecuador (2008-2015). Este modelo permite explicar el contexto ideacional dentro del cual es formulada la política pública a partir de las creencias programáticas de los actores, sus habilidades innatas y sus aprendizajes estratégicos. Del mismo modo, ayuda a comprender el contexto estructural donde se establecen las relaciones entre los actores y el intercambio de sus recursos. La combinación del contexto ideacional y del estructural permite entender cómo se producen los resultados de la política pública.

Para explicar por qué ocurre el cambio de la política pública desde la perspectiva de redes de política es imprescindible entender cómo funciona el poder político dentro de la red; es decir, cómo funciona la «interacción real o potencial entre dos o más actores sociales, en el que uno ejerce mayor control sobre el otro" (Emerson, 1962: 32). Según Knoke, el poder político se ejerce mediante procesos de influencia y de dominación. La influencia opera a través de la persuasión que un actor ejerce sobre otro por medio del intercambio de información, que tiene por objeto cambiar la percepción y las creencias del actor influenciado. La dominación es una relación de poder en la que un actor controla el comportamiento de otro ofreciendo un beneficio o un dańo (Knoke, 1993: 166). Cuando la influencia y la dominación operan simultáneamente en una relación entre dos actores, toma la forma de poder autoritario. En nuestro estudio, esta noción de poder autoritario contribuye a explicar cómo y por qué se da el cambio de la política pública. 
GRÁfico 1.

Modelo ideacional de Kisby

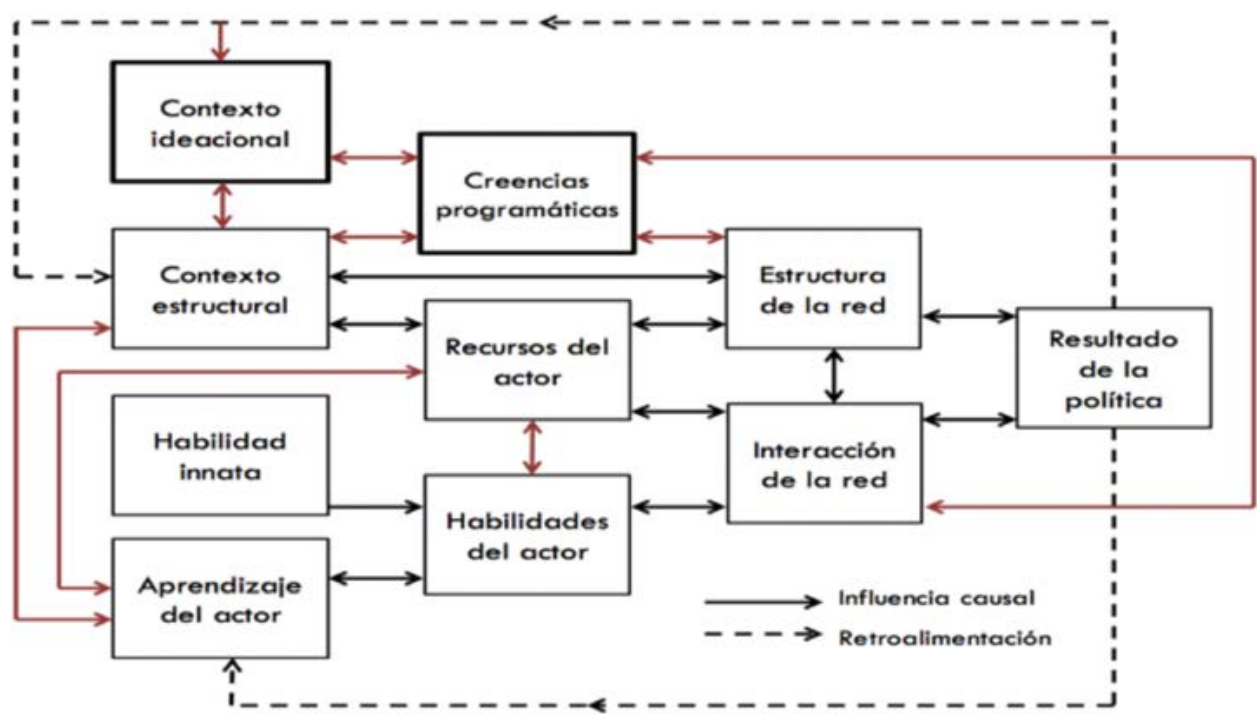

Fuente: traducción propia de Kisby (2007: 84).

En términos metodológicos, hemos operacionalizado el modelo ideacional desde el análisis de redes propuesto por Sanz (2003). Partimos de que las redes contienen «un conjunto de herramientas para conectar el mundo de los actores individuales y colectivos con las estructuras sociales emergentes, que resultan de las relaciones que los actores establecen» (ibid.: 21). Este análisis permite integrar métodos cualitativos y cuantitativos para analizar las interacciones entre las conductas individuales y los patrones de las relaciones en la estructura de la red. Los métodos cualitativos ayudan a agrupar a los actores en torno a sus ideas programáticas, estrategias y recursos, mientras que los métodos cuantitativos, basados en el análisis estadístico de grafos, ofrecen la posibilidad de caracterizar a los actores y sus vínculos.

Para construir las redes de política se ha procedido al rastreo de los actores y sus mediaciones a través del mecanismo de bola de nieve en documentos oficiales (repositorios institucionales), en los Enlaces Ciudadanos 2325 y 378, y archivos de prensa entre 2008 y 2015. También se han realizado entrevistas semiestructuradas a oficiales gubernamentales y a representantes de los GAD para obtener datos sobre las ideas programáticas de los actores en torno a la visión institucional de la política, a las agendas de los actores no institucionales, a los procesos de toma de decisión y al impacto de la autoridad política en el cambio de la política pública.

2. Intervenciones del presidente a través de medios de comunicación públicos y privados. 
A través de estas técnicas, se ha rastreado a los actores en función de la referenciación de unos con otros y de la intensidad de sus relaciones. Cada una de estas relaciones fue asentada y codificada en una base de datos con el fin de mostrar gráficamente la red. Un primer registro se dedicó a los nodos y su tipología: instituciones, instrumentos, hechos, actores particulares, organismos internacionales. Un segundo registro se estableció entre los actores y las ideas programáticas que los cohesionan en redes de política. El resultado de ambos registros se exportó al programa de análisis de redes sociales Gephi 0.82, con el fin de representar mediante una figura la red y de minimizar errores en la información digitalizada.

El análisis cuantitativo de los datos de la red nos permitió caracterizar las relaciones entre los actores por medio de algoritmos estandarizados. El primer algoritmo utilizado fue Force Atlas, que pertenece a la clase de algoritmos de fuerza para dibujar grafos dirigidos, y que permite posicionar los nodos de tal manera que las relaciones entre ellos sean de igual longitud y con la menos cantidad de cruces posibles. Igualmente, se utilizó el algoritmo de expansión para organizar el atributo de nodos con el atributo de nivel y facilitar la identificación de patrones de la red. Por último, con el objeto de conocer la intencionalidad de construcción de la red, la influencia de los actores y su capacidad de intermediar las relaciones en los procesos de toma de decisión se utilizaron las siguientes medidas estadísticas: densidad, centralidad, cercanía e intermediación.

\section{APLICACIÓN DEL MODELO}

El modelo ideacional es aplicado en tres niveles: a) contexto y red; b) estructura y agencia, y c) red y resultados de la política (Marsh y Smith, 2000). El primer nivel explica cómo surgen las redes de política en torno a ideas programáticas; el segundo muestra las relaciones de influencia y dominación que se entretejen entre los actores, en particular el rol de la autoridad política del presidente de la república, y el tercero se ocupa de explicar el resultado de la política como producto de un proceso social y político que ocurre de manera situada en un momento determinado.

\section{Contexto: identificación de las redes}

El contexto permite delimitar el momento y el lugar en el que se analiza el caso y es primordial para identificar a los actores y sus relaciones. El contexto, por tanto, no constituye un elemento de la realidad que se estudia a priori, sino todo lo contrario, es en sí mismo el resultado del reconocimiento de las estructuras exógenas de las redes de política. De este modo, el contexto tiene una relación dialéctica con el surgimiento de las redes de política y, a su vez, es afectado por las redes que se estructuran en dicho momento de la política (ibid.). Por tanto, estudiamos el contexto para identificar tanto las redes de política del contexto estructural, como 
las ideas programáticas que operan en el contexto ideacional (Kisby, 2007). Nos enfocamos en las interacciones entre la estructura de las redes y las ideas que circulan en ellas para estudiar cómo se configuran las redes de política antes, durante y luego del cambio de la política pública.

Hemos identificado tres etapas dentro contexto de la política de ampliación de la cobertura de agua y saneamiento: a) de 2008 a 2013, la norma constitucional establece al agua y saneamiento como un derecho fundamental de la población y se incorpora el componente de subsidios a la financiación de la política; b) en 2014 ocurre el proceso de toma de decisiones sobre sobre la importancia de la continuidad, cantidad y calidad de los servicios, y c) en 2015 tiene lugar la construcción de la Estrategia Nacional de Agua y Saneamiento (ENAS).

\section{Redes 2008-2013}

En 2008, Ecuador cambió la Constitución de la República de Ecuador (CRE) mediante un proceso de Asamblea Constituyente y de referéndum para aprobarla. Este marco regulatorio generó un contexto ideacional fuertemente influenciado por la consideración del agua y saneamiento como un derecho para alcanzar la calidad de vida de la población (CRE arts. 6, 7, 8, 9). Una innovación de la Constitución de 2008 fue la consideración del agua como un patrimonio estratégico de uso público, por lo que se prohibió toda forma de privatización (CRE art. 318) y se ratificó que la competencia de la prestación de servicios de agua y saneamiento corresponde a los GAD (CRE art. 264 y Código Orgánico de Organización Territorial, Autonomía y Descentralizados, art. 55), mientras que la política nacional está a cargo del Gobierno central (CRE art. 318).

Este panorama influyó para que en el año 2012 se conformara la red de política Acuerdo Nacional para el Buen Vivir, donde el presidente y la Asociación de Municipalidades del Ecuador (AME), en representación de los GAD, se comprometieron a trabajar en conjunto. La idea programática de la red constituida dentro del acuerdo nacional es la "calidad de vida" de los beneficiarios. Los propios alcaldes piden «insertarse en temas de mayor perspectiva nacional, demanda que se articula con la oferta del presidente de trabajar en obras que mejoren la calidad de vida de la población" (EACA, 2016). De esta forma se propone la meta de alcanzar una cobertura de $95 \%$ de estos servicios hasta el 2017. Esta meta es incorporada en el Plan Nacional de Desarrollo (Plan Nacional del Buen Vivir), donde se incluye como una de sus políticas el garantizar el acceso universal a estos servicios. Los acuerdos que surgen en esta primera red fortalecen el contexto ideacional de la política, al incluir al agua y saneamiento dentro de la planificación nacional con carácter obligatorio.

Debido al acuerdo entre la AME y el presidente, sumado a las limitaciones presupuestarias de los municipios para financiar obras de este tipo, el presidente manda al Banco del Estado contar con una estrategia de acceso al crédito que incluya un componente no reembolsable. Es así como se consolida la segunda red 
de política Prosaneamiento, cuya idea programática es la «financiación» relacionada con el cumplimiento de las metas nacionales y el cierre de brechas de cobertura. Así se integran varios programas crediticios, incluyendo un alto componente de subvenciones, y en el Enlace Ciudadano 325 se presenta Prosaneamiento, cuyo objetivo es «otorgar subsidios en función de una política pública donde el subsidio mínimo sea del $14 \%$ y el máximo del $92 \%$ (BDE, 2013). La fijación de subsidios se realiza por cantón, en función de los niveles de desnutrición crónica, la pobreza y el déficit para alcanzar el $95 \%$ de prestación de estos servicios básicos (ibid.). Este instrumento consolida la política y produce relaciones en red al involucrar a las instituciones vinculadas con la canalización de recursos, otorgamiento de viabilidades y beneficiarios, estableciendo así un nuevo contexto estructural. Este programa otorga un protagonismo al BDE sobre el resto de actores, reduciendo la problemática del agua y saneamiento a la financiación, y dejando de lado temas como continuidad, cantidad y calidad de los servicios. El contexto ideacional se enmarca alrededor de las dificultades de los GAD para acceder a líneas de crédito debido a sus bajos cupos de endeudamiento.

La tercera red de política surgió debido a los problemas que enfrentan los GAD y se creó la Alianza Estratégica Municipal por el Agua, cuya idea programática era alcanzar una "gestión eficiente» mediante el establecimiento de procesos más ágiles y del fortalecimiento de capacidades para el acceso al crédito y prestación de estos servicios. Esta red es liderada por la AME y en ella participan más de sesenta municipios con la finalidad de recibir asistencia técnica por parte de los GAD con las mejores empresas de agua y saneamiento del país. En las elecciones de febrero de 2014, los alcaldes de los GAD que prestaban asistencia técnica no fueron reelegidos, y hubo un cambio de autoridades en el resto de municipios. Las alcaldías de las ciudades más grandes del país fueron ocupadas por movimientos de oposición al partido de gobierno, presentando una amplia gama de ideologías y tendencias políticas. Como consecuencia de estos resultados electorales, secciones de esta alianza se desintegraron, lo que ocasionó la disolución de la red.

\section{Redes 2014}

En términos estructurales, la SENAGUA asumió la dirección de la política de agua y saneamiento en lugar del MIDUVI. Además, en términos ideacionales se fortaleció el cumplimiento de las metas nacionales y el cierre de brechas de cobertura en el marco de la Estrategia para la Igualdad y la Erradicación de la Pobreza (SENPLADES, 2014d). Con este telón de fondo, la Secretaría Técnica para la Erradicación de la Pobreza (SETEP), junto con la Secretaría Nacional de Planificación y Desarrollo (SENPLADES) lideró la cuarta red de política a través de una Propuesta Interinstitucional para la ampliación de la cobertura de estos servicios y movilizando sus ideas en relación con la "cobertura, gestión y calidad» (SENPLADES, 2014b). En torno a esta propuesta se crearon redes de política más amplias que incorporan 
entidades relacionadas con la planificación nacional, el sector social y el sector financiero. Por tanto, el contexto estructural se modificó al incluir a más actores en comparación con el período 2008-2013. Esta red planteó la necesidad de reducir los tiempos para la aprobación de los proyectos de inversión y establecer facilidades crediticias para los GAD con las mayores carencias. El BDE se opuso a esta red, sosteniendo que los GAD deben cumplir todas las condiciones para el acceso al crédito, entre las que se destaca su disponibilidad de cupo de endeudamiento y su capacidad de pago.

En el mismo año, se conformó la quinta red de política Cambio de Metas. Esta red surgió por la «imposibilidad de cumplimiento de las metas» anteriormente establecidas, debido a la poca capacidad instalada en el país y a que los tiempos de construcción iban a sobrepasar el límite del año 2017. Por estas razones, se modificó a la baja la meta de cobertura de agua a nivel nacional, y se pasó de una meta de cobertura del $95 \%$ a una del $83 \%$ (SENPLADES, 2014c). Este cambio de metas fue liderado por SETEP, SENPLADES y el BDE, desplazando el rol de SENAGUA como rectora de la política de agua y saneamiento.

Finalmente, se creó la sexta red de política en junio de 2014 entre el BDE, el Ministerio de Economía y Finanzas (MEF) y el presidente. Esta nueva red propuso como idea programática la «eliminación de los subsidios» destinados con la financiación de competencias que no le correspondan al Gobierno central. Esta decisión fue comunicada por el presidente a través del Enlace Ciudadano 378, en donde presentó un análisis histórico de la concesión de subsidios desde el BDE. En el periodo 2000-2006 (anterior a su gobierno) se concedieron 434 millones de dólares, con un total de $10 \%$ en subsidios (41 millones de dólares), mientras que durante su gobierno (2007-2014) se concedieron 3540 millones de dólares, con un total del $46 \%$ en subsidios (1625 millones de dólares) para este tipo de competencias. Esto implica, en palabras del presidente, que «no existe verdadera autonomía, porque la mayor parte de la financiación para los municipios y prefecturas llega a través de transferencias del Gobierno central [...] yo no puedo estar pagando competencias que no son nuestras, mientras yo no puedo cumplir con nuestras competencias y obligaciones»(BDE, 2014). Con este argumento, el presidente decidió fundamentar su propuesta de eliminar los subsidios a los GAD y anunció una reingeniería para la ampliación al doble de tiempo para los plazos de los GAD y para triplicar su capacidad de endeudamiento, a modo de compensación por la eliminación de los subsidios a algunas líneas de crédito. Con estas declaraciones, se anunciaron los cambios para el acceso a créditos otorgados por el BDE, que terminan modificando la política de agua y saneamiento, al cambiar las características de Prosaneamiento (tabla 2).

De igual manera, el presidente recomendó el fortalecimiento del sistema impositivo de los municipios para hacer sostenible la prestación de sus competencias a través de la actualización de los catastros y la recaudación del impuesto predial. La intervención del presidente es un ejemplo de cómo las «decisiones externas pueden moldear directamente elementos del diseño de política cuando el núcleo decisional se desplaza 
TABLA 2.

Condiciones de Prosaneamiento

\begin{tabular}{|c|c|c|}
\hline Características & Condiciones iniciales & Condiciones finales \\
\hline Preinversión & $\begin{array}{l}\text { Financiación no reembolsable del } \\
100 \%\end{array}$ & $\begin{array}{l}\text { Financiación no reembolsable del } \\
60 \%\end{array}$ \\
\hline Inversión & $\begin{array}{l}\text { Financiación no reembolsable en } \\
\text { promedio de } 46 \%\end{array}$ & $\begin{array}{l}\text { Financiación } 100 \% \text { reembolsable } \\
\text { Recálculo para triplicar la capacidad } \\
\text { de endeudamiento en relación de } \\
2013\end{array}$ \\
\hline Requisitos & $\begin{array}{l}\text { Viabilidad técnica emitida por } \\
\text { SENAGUA. } \\
\text { Certificación ambiental emitida por } \\
\text { el Ministerio del Ambiente (MAE) } \\
\text { en función del Texto Unificado de } \\
\text { Legislación Ambiental Secundaria } \\
\text { (TULAS) }\end{array}$ & $\begin{array}{l}\text { Actualización catastral del GAD. } \\
\text { Financiación } 50 \% \text { reembolsable. } \\
\text { Viabilidad técnica emitida por } \\
\text { SENAGUA. } \\
\text { Certificación ambiental emitida por } \\
\text { el MAE en función del TULAS }\end{array}$ \\
\hline Tiempos & Hasta 7 ańos en promedio & Hasta 15 años \\
\hline
\end{tabular}

Fuente: elaboración propia.

a un nivel superior del subsistema o a una arena de acción exterior» (Real Dato, 2009: 136). Es así como la decisión del presidente modificó el contexto, al eliminar los incentivos a la inversión a través de Prosaneamiento y con ello también alteró las relaciones entre la agencia y la estructura de la red.

\section{Red 2015}

A finales de 2014, SENAGUA lideraba la construcción de la ENAS, bajo la idea programática de contar con orientaciones a las intervenciones e inversiones desde «una perspectiva sectorial». El objetivo era proporcionar una "guía oficial para el sector en su intención de lograr una cobertura total de los servicios de agua y saneamiento en todo el territorio nacional en los próximos años y dar así cumplimiento tanto a las metas del Plan Nacional del Buen Vivir como de la Estrategia de Erradicación de la Pobreza» (SENAGUA, 2015: 9). Esta séptima red de política permite la articulación de organismos internacionales, Gobiernos locales por medio de la AME y de las Juntas Administradoras de Agua Potable y Saneamiento (JAAPS) que son prestadores comunitarios, y de actores del Ejecutivo relacionados con la planificación, asignación de recursos, regulación y control.

Para sintetizar las redes que se configuran en el contexto de la política, presentamos a continuación el detalle de los nodos de las redes en cada uno de los tres momentos del contexto, como paso previo a la representación de los grafos de las redes de la política de ampliación de la cobertura de agua y saneamiento 2008-2015. 
TABLA 3.

AfILIACión DE NODOS POR ETAPA

\begin{tabular}{|c|c|c|c|c|}
\hline Tipo de nodo & Descripción & 2008-2013 & 2014 & 2015 \\
\hline \multirow{23}{*}{ Institución } & Asociación de Municipalidades del Ecuador (AME) & 1 & 1 & 1 \\
\hline & Agencia de Regulación y Control del Agua (ARCA) & 0 & 0 & 1 \\
\hline & Banco del Estado (BdE) & 1 & 1 & 1 \\
\hline & Consejo Nacional de Competencias (CNC) & 0 & 0 & 1 \\
\hline & Empresa Pública del Agua (EPAGUA) & 0 & 0 & 1 \\
\hline & Empresa Pública de Quito (EP-Q) & 1 & 0 & 0 \\
\hline & Empresa Pública de Cuenca (EP-C) & 1 & 0 & 0 \\
\hline & Empresa Pública de Ambato (EP-A) & 1 & 0 & 0 \\
\hline & Empresa Pública de Ibarra (EP-I) & 1 & 0 & 0 \\
\hline & Gobiernos Autónomos Descentralizados (GAD) & 1 & 1 & 1 \\
\hline & Instituto Nacional de Estadística y Censos (INEC) & 0 & 0 & 1 \\
\hline & Instituto Nacional de Preinversión (INP) & 0 & 1 & 0 \\
\hline & $\begin{array}{l}\text { Juntas Administradoras de Agua y Saneamiento } \\
\text { (JAAPS) }\end{array}$ & 1 & 1 & 1 \\
\hline & Ministerio del Ambiente (MAE) & 1 & 1 & 1 \\
\hline & Ministerio Coordinador de Desarrollo Social (MCDS) & 0 & 1 & 1 \\
\hline & $\begin{array}{l}\text { Ministerio Coordinador de Sectores Estratégicos } \\
\text { (MCSE) }\end{array}$ & 0 & 0 & 1 \\
\hline & Ministerio de Economía y Finanzas (MEF) & 1 & 1 & 1 \\
\hline & $\begin{array}{l}\text { Ministerio de Desarrollo Urbano y Vivienda } \\
\text { (MIDUVI) }\end{array}$ & 1 & 0 & 0 \\
\hline & Ministerio de Salud Pública (MSP) & 0 & 1 & 0 \\
\hline & Secretaría Nacional del Agua (SENAGUA) & 0 & 1 & 1 \\
\hline & $\begin{array}{l}\text { Secretaría Nacional de Planificación y Desarrollo } \\
\text { (SENPLADES) }\end{array}$ & 1 & 1 & 1 \\
\hline & $\begin{array}{l}\text { Secretaría Técnica para la Erradicación de la Pobreza } \\
\text { (SETEP) }\end{array}$ & 0 & 1 & 1 \\
\hline & Secretaría Nacional de Gestión de la Política (SGP) & 0 & 1 & 1 \\
\hline \multirow{5}{*}{ Instrumentos } & $\begin{array}{l}\text { Código Orgánico de Organización Territorial, } \\
\text { Autonomía y Descentralización (COOTAD) }\end{array}$ & 1 & 1 & 1 \\
\hline & Estrategia Nacional de Agua y Saneamiento (ENAS) & 0 & 0 & 1 \\
\hline & $\begin{array}{l}\text { Estrategia Nacional para la Igualdad y la } \\
\text { Erradicación de la Pobreza (ENIEP) }\end{array}$ & 0 & 0 & 1 \\
\hline & Programa Prosaneamiento & 1 & 1 & 1 \\
\hline & Texto Unificado de Legislación Ambiental (TULAS) & 1 & 1 & 1 \\
\hline
\end{tabular}




\begin{tabular}{|c|c|c|c|c|}
\hline Tipo de nodo & Descripción & 2008-2013 & 2014 & 2015 \\
\hline \multirow{5}{*}{$\begin{array}{l}\text { Organismo } \\
\text { Internacional }\end{array}$} & Banco Alemán (KfW) & 1 & 1 & 1 \\
\hline & Banco Interamericano de Desarrollo (BID) & 1 & 1 & 1 \\
\hline & Banco Mundial (BM) & 1 & 1 & 1 \\
\hline & Corporación Andina de Fomento (CAF) & 1 & 1 & 1 \\
\hline & Fondo de Cooperación Española (FCE) & 0 & 0 & 1 \\
\hline \multirow{4}{*}{ Hecho } & Acuerdo Nacional por el Buen Vivir(ANBV) & 1 & 0 & 0 \\
\hline & Alianza Estratégica Municipal por el Agua(AEMA) & 1 & 0 & 0 \\
\hline & Cambio Metas PNBV & 1 & 1 & 0 \\
\hline & Propuesta interinstitucional(Propuesta) & 0 & 1 & 0 \\
\hline $\begin{array}{l}\text { Actor } \\
\text { Particular }\end{array}$ & Presidente & 1 & 1 & 0 \\
\hline
\end{tabular}

Fuente: elaboración propia.

GRÁFICO 2.

REDES DE LA PRIMERA ETAPA

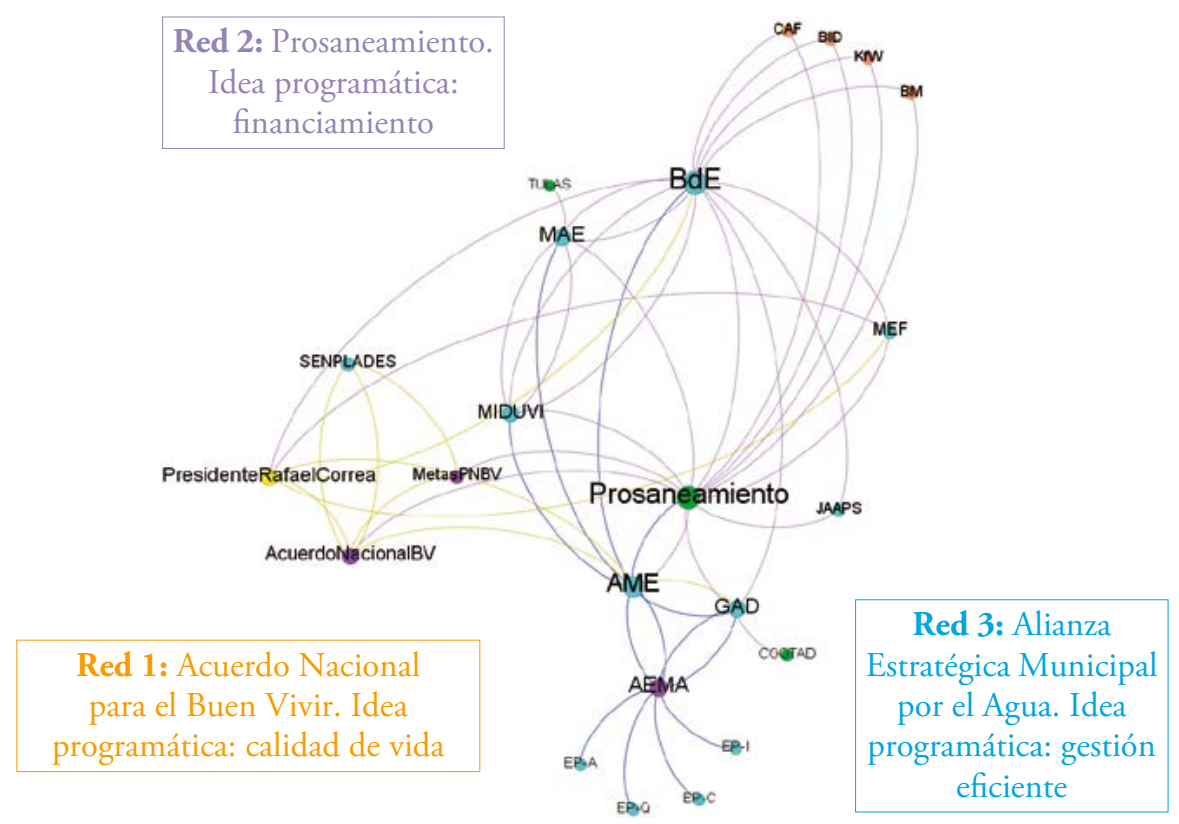

Tipo de nodo

Institución Instrumento Organismos internacionales Hecho Actor particular

Fuente: elaboración propia. 
Gráfico 3.

REDES DE LA SEGUNDA ETAPA

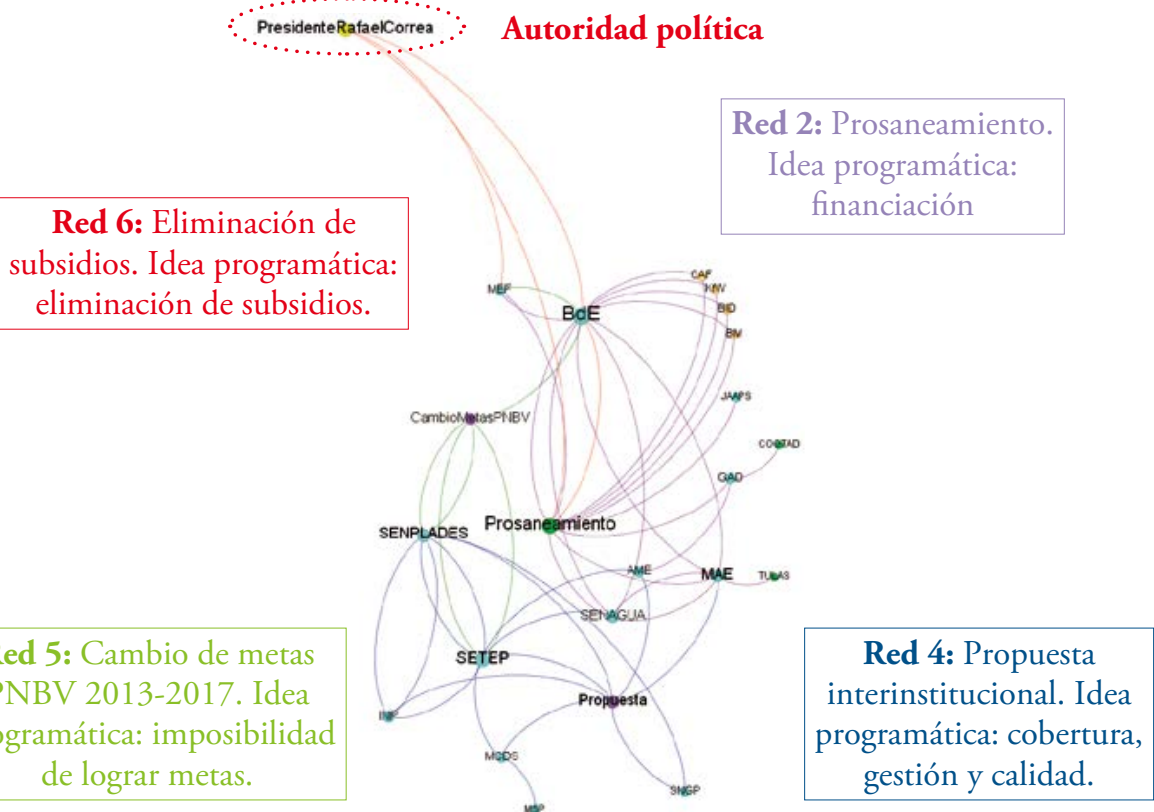

Tipo de nodo

Institución Instrumento Organismos internacionales Hecho Actor particular

Fuente: elaboración propia.

La primera etapa del contexto ideacional estuvo fuertemente influenciada por la consideración de que el agua y el saneamiento son un derecho fundamental para alcanzar mejor calidad de vida de la población y el cumplimiento de otros derechos tales como salud, ambiente sano, hábitat y vivienda. Una innovación de la Constitución de 2008 fue considerar el agua como un "patrimonio estratégico de uso público", lo que prohíbe toda forma de su privatización. Este panorama influyó para que en el año 2012 se conformara la red de política en la que el presidente Rafael Correa y la AME, en representación de los GAD, se interesaran por trabajar en conjunto, para alcanzar una cobertura del $95 \%$ de estos servicios en 2017. Con ello, se fortaleció la idea de incluir el agua y el saneamiento dentro de la planificación nacional de manera obligatoria. Este acuerdo entre la AME y el presidente comprometió al BDE a contar con una estrategia de acceso al crédito que incluyera un porcentaje importante de fondos no reembolsables. A su vez, esto permitió la consolidación de la red de política en torno a Prosaneamiento, más enfocada al cumplimiento de las metas nacionales y a la disminución de las brechas de cobertura gracias a programas crediticios con un alto componente de subvenciones. Las dificultades para el acceso al crédito de los 
GAD fueron identificadas y canalizadas por la AME, reconociendo que las más importantes eran los bajos cupos de endeudamiento establecidos y los largos tiempos para el acceso a la financiación. Esto impulsó la red de la Alianza Estratégica Municipal por el Agua, que tiene como idea fuerza el alcanzar una gestión más eficiente.

En la segunda etapa, el contexto ideacional promovió las ideas que ponen de relieve el cumplimiento de las metas nacionales y el cierre de las brechas de cobertura. Como ya hemos anotado, se produjo un cambio en la institución que asume la dirección de la política y se favoreció la red que apoya la "propuesta interinstitucional» para la ampliación de estos servicios. Esto permitió una mayor incorporación de las entidades relacionadas con la planificación nacional, el sector social y el sector financiero. Sin embargo, una nueva intervención del presidente de la república fuera de los consensos promovidos por el Ejecutivo de defender el agua como un derecho constitucional y de la planificación nacional subvencionada por el Gobierno central, produjo el cambio de primer orden de la política pública. Esta intervención externa, sin diálogo con los actores, transformó el proceso de toma de decisiones y reformuló los instrumentos de la política, produciendo un importante cambio en las ideas programáticas de la política.

\section{GrÁFICO 4.}

REDES DE LA TERCERA ETAPA

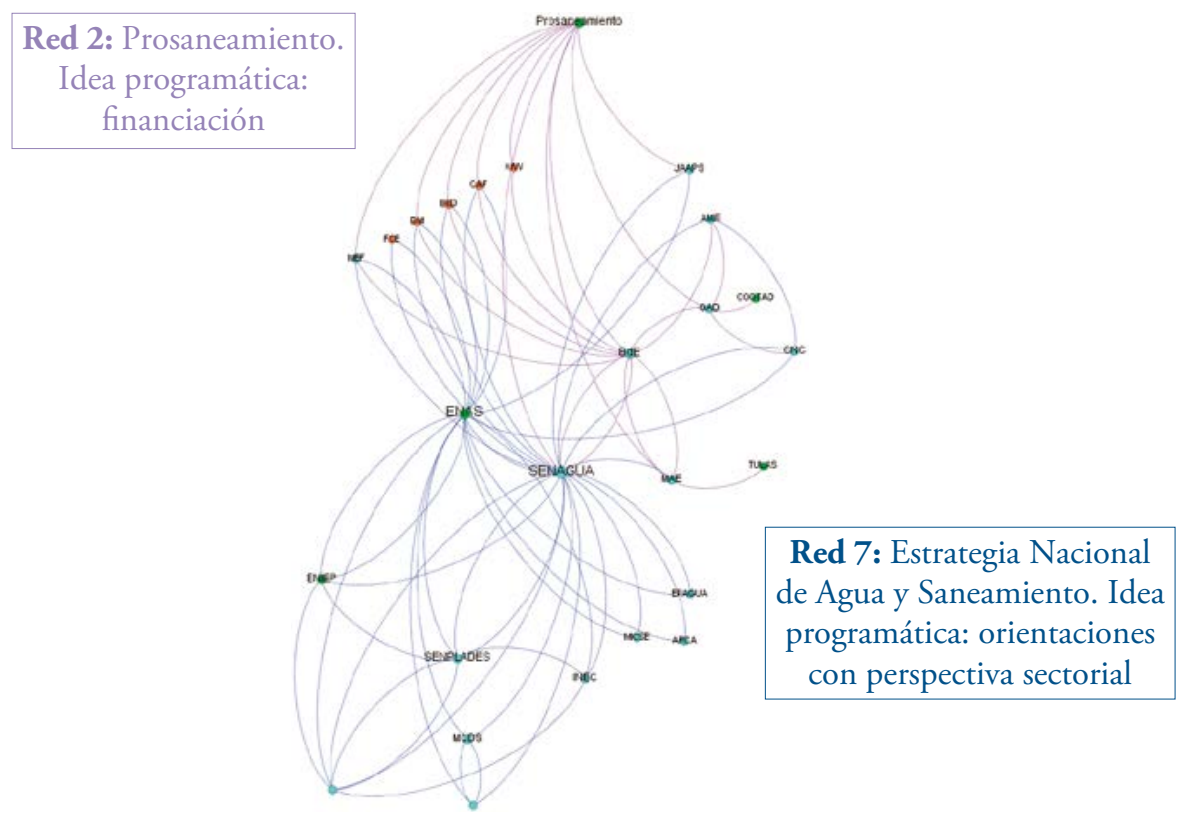

Tipo de nodo

Institución Instrumento Organismos internacionales Hecho Actor particular

Fuente: elaboración propia. 
La intervención del presidente alteró el acuerdo de la red de otorgar créditos subsidiados y reemplazó la idea de financiación por la de eliminación de subsidios a los GAD, utilizando al BDE como uno de los actores ejecutores de la propuesta presidencial. Esto hizo que la entidad rectora de la política, SENAGUA, asumiera un rol secundario en la toma de decisiones y que se redujera la viabilidad técnica para la construcción de infraestructura a nivel nacional.

La tercera etapa evidencia cómo desaparecerieron las redes de política que se habían consolidado antes de la intervención directa del presidente y cómo se reconfiguró la política nacional dando paso a una política sectorial. También muestra cómo la SENAGUA se esforzó por diseñar un nuevo instrumento de política, cuando la política pública quedó huérfana de orientaciones que guiaran las inversiones e intervenciones que requiere el sector. Esta es la razón por la cual se construyó la ENAS, cuyo objeto era establecer criterios para alcanzar la universalización del agua y saneamiento en el Ecuador.

\section{Estructura y agencia de las redes de politica}

Las redes de política institucionalizan los sistemas de creencia de los actores y, a partir de su estructura, pueden incluso configurar comportamientos y actitudes. «Las redes no son neutras, por el contrario, inscriben la distribución del poder y de los conflictos del pasado que han incidido sobre los resultados presentes de la política» (Albornoz, 2015: 175). En este sentido, analizamos la estructura y la agencia de las redes de política dentro de una relación simétrica. Por una parte, nos ocupamos de la visión del mundo que mantienen los actores a modo de limitación estructural y, por otra, de las preferencias de los actores, sus construcciones ideacionales y las negociaciones que realizan dentro de un contexto estructurado.

Para explicar la incidencia de las preferencias y comportamientos individuales de los actores y de sus comportamientos colectivos, usamos las ideas programáticas como elemento estructurador de las redes de política. Aplicamos el análisis de grafos para mostrar cómo los actores interactúan entre sí, cómo se producen las negociaciones entre las redes el momento del cambio de la política, y de qué modo actúa la autoridad política para reorganizar las relaciones de poder entre las redes. Las medidas estadísticas utilizadas en este análisis de redes son: densidad, centralidad, cercanía y autoridad.

Utilizamos la medida de densidad para conocer si la red ha sido construida de manera intencional. La densidad de la red es baja, apenas alcanza un 10\%, lo que nos permite confirmar que la red es construida de manera intencional desde los actores estatales. La centralidad de la red mide los nodos con el mayor número de relaciones tanto de entrada como de salida. Encontramos que desde el 2008 hasta el 2014 sobresalen el BDE (15) con su instrumento de financiamiento Prosaneamiento (14), mientras que en el 2015 tiene mayor centralidad SENAGUA (24) con el instrumento de planificación ENAS (18). Esta medida muestra cuáles son los actores con mayor influencia en la implementación de la política: el BDE y SENAGUA con sus respectivos instrumentos. 


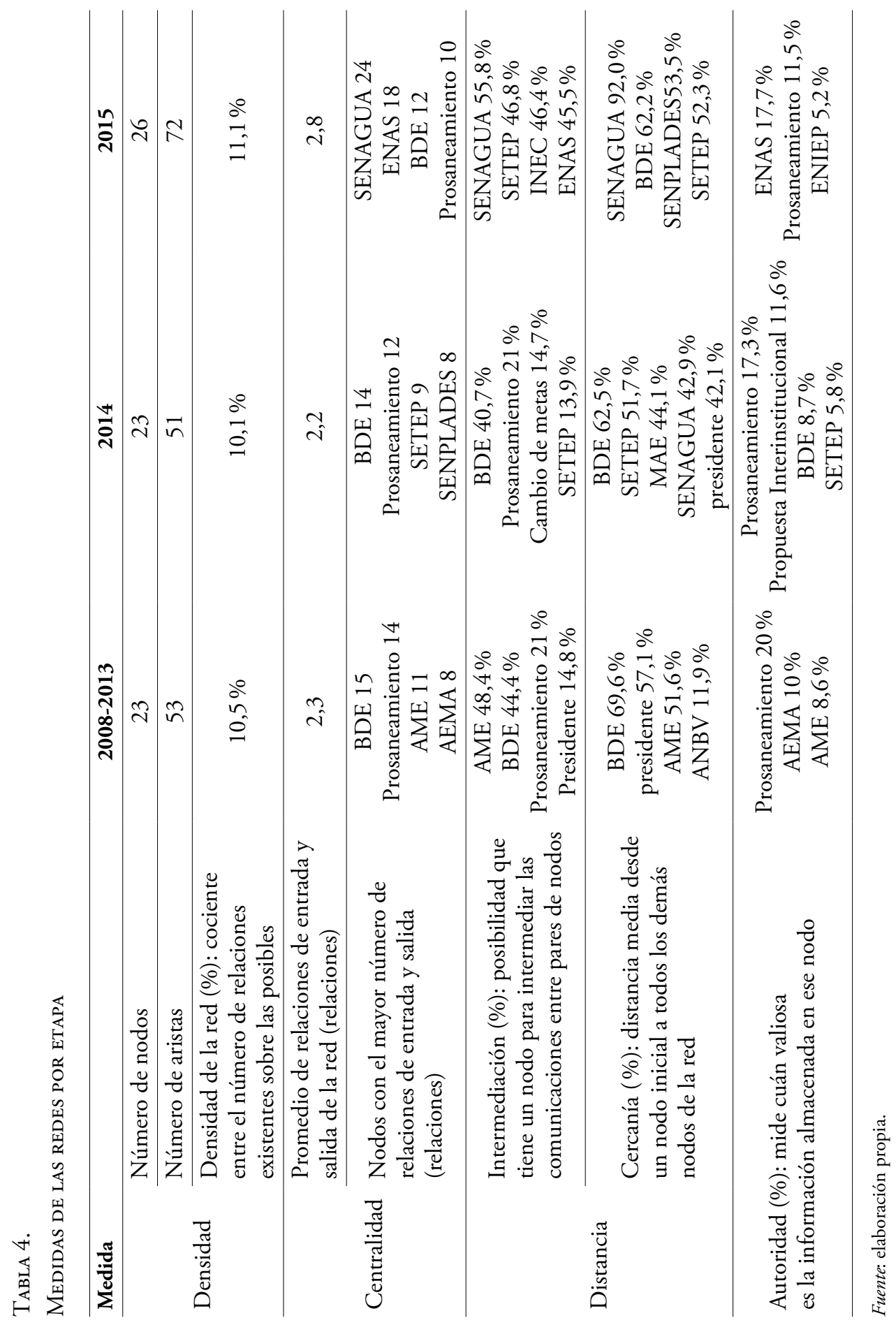


La intermediación es una medida que permite identificar a los actores que se convierten en los puntos de paso obligado de otros actores dentro de la red. Por ejemplo, entre 2008 y 2013 es la AME (como representante de los GAD) el actor que más intermediaciones realiza (48,4\%), seguida por el BDE (44,4\%). En 2014 se mantiene el BDE con mayor porcentaje de intermediación (40,7\%), seguido de Prosaneamiento (21\%). En el 2015, una vez que se da el cambio de la política pública, los nuevos intermediarios son SENAGUA $(55,8 \%)$ y SETEP $(46,8 \%)$, que actúan como articuladores de la construcción de la Estrategia Nacional de Agua y Saneamiento. Sin embargo, el BDE y Prosaneamiento son los nodos con mayor habilidad para agrupar otros actores y para mantener la mayor cantidad de relaciones dentro de la red a lo largo del cambio de la política pública. En particular, el banco es uno de los actores más fuertes intermediando relaciones con entidades del Gobierno central, Gobiernos locales y organismos internacionales.

La cercanía es una medida que permite conocer cuáles son los nodos de mayor influencia a lo largo de la transformación de la política pública, pues mide la menor distancia media hacia el resto de nodos de la red. Encontramos que el BDE mantiene un alto valor de cercanía (sobre el 62\%) en todos los momentos del contexto de la política pública. El presidente le sigue con un $57,1 \%$ y un $42,1 \%$, respectivamente, entre 2008 y 2014, y la AME con un 51,6\% entre 2008 y 2013. En el 2014 los nodos con alta cercanía son la SETEP $(51,7 \%)$ y el Ministerio del Ambiente del Ecuador (MAE) $(41,1 \%)$, hasta que en 2015 , con el cambio de la política pública, SENAGUA se convierte en el actor con la mayor influencia con un $92 \%$ de cercanía, mientras que SENPLADES adquiere relevancia con el 53,5\%.

Finalmente, la medida de autoridad mide cuán valiosa es la información almacenada en un nodo. Así, Prosaneamiento es el nodo que mayor autoridad alcanza con el $20 \%$, seguido de la Alianza Estratégica Municipal por el Agua con un $10 \%$. Prosaneamiento mantiene su medida de autoridad (17,3\%) en el 2014 junto con la Propuesta Interinstitucional $(11,6 \%)$ promovida por SENPLADES y SETEP. Prosaneamiento no pierde autoridad $(11,5 \%)$ en el 2015 como el principal programa de financiamiento de los GAD para la construcción de obras. Sin embargo, también adquieren autoridad en este año la ENAS (17,7\%), encargada de proporcionar directrices para el sector de agua y saneamiento, y la ENIEP $(5,2 \%)$ como el principal instrumento de política pública para el combate a la pobreza y la desigualdad.

Dentro del análisis cuantitativo, el presidente no logra en ningún momento un rol central, dadas sus escasas relaciones de entrada y salida con otros nodos; esto lo coloca como un elemento exógeno dentro del contexto de la red. Sin embargo, ejerce autoridad política y transforma la política pública a través de relaciones de influencia y dominación, ejerciendo el poder político necesario para favorecer el cambio de primer orden de la política. El análisis de grafos ilumina el fenómeno del mecanismo de impacto exógeno, como un fenómeno externo a las relaciones que se establecen entre los actores de las redes de política.

Para explicar cómo la autoridad del presidente es capaz de transformar la red y de generar el cambio de la política pública, recurrimos a la propuesta de Knoke sobre 
cómo funciona el poder político dentro de las interacciones entre los actores de una red de política (1993). Puesto que dentro de nuestro análisis cuantitativo son otros los actores, y no el presidente, los que alcanzan mayores medidas de intermediación, cercanía y autoridad, es fundamental explicar las relaciones de poder que se desarrollan dentro de la red para mostrar cómo la autoridad política funciona como un factor exógeno de la red capaz de cambiar la política de agua y saneamiento. De esta forma, vemos cómo el presidente tiene la capacidad de tomar decisiones que moldean el núcleo de decisiones del subsistema en el que la política se diseña y de sacar el proceso de toma de decisiones fuera de la arena de acción en donde está ubicado (Real Dato, 2009). Así, la autoridad política se constituye en la restricción más importante del contexto de la política pública (Marsh y Smith, 2000).

Para explicar cómo se realiza el cambio de la política pública se ha procedido a analizar las distintas agendas de las redes de política dentro de la trayectoria de la política pública. Estas agendas nos permiten contrastar con mayor precisión cuáles son las relaciones de influencia y dominación que ejercen los distintos actores para lograr el poder político necesario para el cambio de la política pública. En este sentido, rastreamos las ideas programáticas contenidas en las agendas de las redes de política y mapeamos las negociaciones que logran estabilizar unas ideas sobre otras. Por ejemplo, vemos que el agua se asocia a calidad de vida dentro del Acuerdo Nacional para el Buen Vivir; al financiamiento desde Prosaneamiento; a la gestión eficiente dentro de la Alianza Estratégica Municipal por el Agua; a la cobertura, gestión y calidad en la Propuesta Institucional; a la dificultad de alcanzar las metas propuestas para el $2017 \mathrm{y}$, finalmente, a la eliminación de subsidios desde la postura del presidente. Sin embargo, son las relaciones de influencia y dominación las que nos permiten explicar cómo las agendas son negociadas y quiénes imponen sus ideas programáticas.

Entre 2008 y 2013, los actores mantienen relaciones funcionales sustentadas en las responsabilidades asignadas a partir del cambio constitucional de 2008. De esta forma, el BDE ofrece algunas líneas de crédito aisladas y canaliza los recursos negociados con el MEF y con organismos internacionales. El MAE y el MIDUVI se encargan de la revisión de los proyectos a nivel técnico y ambiental, respectivamente, y el MIDUVI ejerce un rol débil, sin asumir la rectoría de esta política. Por otro lado, la AME mantiene relaciones de influencia con el presidente para la suscripción del Acuerdo Nacional para el Buen Vivir, con las empresas de saneamiento más importantes del país para la generación de la Alianza Estratégica Municipal por el Agua, y con la SENPLADES para la incorporación de las metas basadas en la ampliación de la cobertura de estos servicios dentro del Plan de Desarrollo.

En el 2014, la SETEP y la SENPLADES generan alianzas con varios actores en torno a la Propuesta interinstitucional, basada en la idea programática de cobertura, gestión y calidad. Para ello, utilizan la persuasión logrando agrupar a la AME como representante de los GAD, al Ministerio Coordinador de Desarrollo Social (MSP) y al Ministerio de Salud Pública (MSP) del sector social, y a SENAGUA y al MAE como actores involucrados en la revisión de los proyectos de agua y saneamiento. Incluso incorporan a la Secretaría Nacional de Gestión de la Política con la finalidad de elevar 
la propuesta interinstitucional. Igualmente, se establecen relaciones entre la SETEP, el Banco del Estado y el MEF, dentro del trabajo técnico desarrollado para el cambio de las metas nacionales en el marco de los limitantes para su cumplimiento.

En el 2015 se mantienen las relaciones funcionales entre los actores involucrados en la política. No obstante, lo novedoso en este año es la construcción de la ENAS, que genera relaciones de influencia para adherir un mayor número de actores bajo la idea programática de contar con orientaciones desde la perspectiva sectorial. La ENAS permite la articulación de organismos internacionales, Gobiernos locales por medio de la AME y JAAPS, y actores del Ejecutivo relacionados con la planificación, asignación de recursos, regulación y control.

Las redes de política manejan relaciones de influencia entre sí para estabilizar la implementación de la política. La figura del presidente funciona como un mecanismo de impacto exógeno a la red produciendo el cambio de la política. El poder autoritario (Knoke, 1993: 166) del presidente hace que las redes de política se subordinen jerárquicamente al ámbito de acción de la presidencia al combinar elementos de influencia y de dominación. Primero, promueve el otorgamiento de créditos subsidiados a los GAD a través del Banco del Estado, para luego instaurar la eliminación del componente no reembolsable. Este giro provoca el cambio de la política pública al restringir los recursos que los GAD contaban para ejecutar la política pública.

\section{Red y resultado}

Los productos de la política reflejan una relación dialéctica entre la estructura de la red y sus interacciones, y no constituye la simple suma de los efectos de las estructuras y sus agentes (Marsh y Smith, 2000). "Considerando que no existe un vínculo causal unidireccional entre redes y resultados, las redes pueden afectar los resultados de las políticas, ya que constituyen la estructuración de los conflictos del pasado y el poder organizacional del presente. En este nivel los aprendizajes estratégicos se convierten en un mecanismo de acción muy efectivo» (Albornoz, 2015: 177).

Analizamos los elementos que influyen para alcanzar el cambio de la política, entendiendo la transformación de la política pública como resultado de un proceso social y político que ocurre en un período de tiempo determinado (Rayner, 2009: 83). $\mathrm{Al}$ tiempo que explicamos los efectos que este resultado tiene sobre los actores, sus posiciones y procesos de toma de decisiones. Igualmente analizamos la evolución de las aprobaciones crediticias para el sector de agua y saneamiento desde el BDE, con la finalidad de determinar si después del cambio de política existe alguna variación en cuanto a la participación de este sector frente al total de sectores financiados.

Nuestro caso tiene las características de un cambio incremental de primer orden propuesto por Hall (1993), ya que se limita a la calibración del instrumento de la política pública siguiendo un proceso evolucionario. Se trata también de un cambio no lineal en el que la política tiene un comportamiento irregular e inesperado, pues no sigue una secuencia preestablecida (Capano, 2009: 12). La intervención del presidente 
y el uso de sus relaciones de influencia y dominación sobre las redes de política se explica a través del uso del poder autoritario (Knoke, 1993) y de la lógica de lo adecuado (Real Dato, 2009) para eliminar el componente no reembolsable de Prosaneamiento, con la finalidad de que los GAD sean responsables de sus propias competencias. Debemos aclarar que este mecanismo exógeno hace referencia a un actor en particular y no a una institución. Por tanto, cuando nos referimos al presidente no hablamos de la presidencia como institución ni como un equipo que promueve el cambio de la política pública. Hacemos alusión a un actor en particular, que es vocero de sí mismo y es capaz de cambiar de opinión e imponerla jerárquicamente a un subsistema de política.

Siguiendo el modelo ideacional de Kisby (2007) se determina que el resultado de política tiene afectaciones en el contexto estructural, en la estructura y en las interacciones de la red. Por ejemplo, la idea de financiamiento (sin el componente no reembolsable) da cabida al surgimiento del ENAS, que cambia la estructura de la red, otorgándole elementos de gobernanza frente a la política centralizada que se impone luego de la eliminación de subsidios desde el presidente. $\mathrm{Y}$ al tratarse de un cambio de primer orden, el resultado del cambio podría ser reversible. La estructura de las redes se modifica debido a la intervención del presidente, que anula los consensos generados e influye en la conformación de la red de la ENAS, basada en la idea programática de plantear los lineamientos para las intervenciones e inversiones desde una perspectiva sectorial.

El cambio de política tiene implicaciones en las interacciones de las redes, ya que establece que las decisiones de financiamiento pasen necesariamente por el presidente, mientras que las decisiones relacionadas con la planificación y gestión recaigan en

TABLA 5.

Problemas epistemológicos Y Teóricos Del CAMbio de POlítica de AgUA Y SANEAMiento

\begin{tabular}{cll}
\hline \multirow{2}{*}{$\begin{array}{c}\text { Problemas } \\
\text { epistemológicos }\end{array}$} & Motores del cambio & $\begin{array}{l}\text { Factores exógenos sobre el subsistema de } \\
\text { política: intervención de la autoridad política }\end{array}$ \\
\cline { 2 - 3 } & $\begin{array}{l}\text { Forma de progresión del } \\
\text { evento }\end{array}$ & No lineal: comportamiento inesperado \\
\cline { 2 - 3 } & Dinámica de desarrollo & Evolucionaria \\
\hline \multirow{3}{*}{$\begin{array}{c}\text { Problemas } \\
\text { teóricos }\end{array}$} & $\begin{array}{l}\text { Definición del objeto del } \\
\text { cambio }\end{array}$ & Alteración del instrumento Prosaneamiento \\
\cline { 2 - 3 } & Tipo de cambio & Primer orden (incremental) \\
\cline { 2 - 3 } & Variables explicativas & $\begin{array}{l}\text { Intervención autoridad política (cambio en la } \\
\text { primacía de ideas programáticas) }\end{array}$ \\
\cline { 2 - 3 } & Dilema estructura/agencia & $\begin{array}{l}\text { Agencia del presidente (comportamiento } \\
\text { individual) }\end{array}$ \\
\cline { 2 - 3 } & Resultado & \begin{tabular}{l} 
Reversible \\
\hline
\end{tabular}
\end{tabular}

Fuente: elaboración propia. 
SENAGUA — para la emisión de lineamientos de política — y en el BDE — para el manejo de Prosaneamiento-. En relación con los aprendizajes estratégicos de los actores de la red, la alteración de la política incide en que el BDE incorpore mecanismos de compensación para incentivar el acceso a préstamos por parte de los GAD, tales como el incremento de cupos de endeudamiento y la ampliación de plazos para el pago de créditos, y para que SENAGUA inicie el proceso de construcción de la ENAS.

Con la finalidad de complementar el análisis del cambio de política pública, se explora la evolución de las aprobaciones del BDE relacionadas con el sector de agua y saneamiento. $\mathrm{Al}$ respecto, es importante señalar que las aprobaciones hacen referencia a la aceptación de financiación de obras del Banco, para lo cual es necesario que los GAD cuenten con las viabilidades técnicas y ambientales emitidas por SENAGUA y MAE, respectivamente. También deben contar con las viabilidades económica y financiera emitidas por el Banco, consistente en un análisis de riesgo y en el establecimiento de condiciones conforme a la capacidad de endeudamiento. En este sentido, las aprobaciones realizadas durante un año determinado son el reflejo de un trabajo previo cuyo proceso abarca la solicitud del préstamo, la elaboración del proyecto de inversión, el otorgamiento de viabilidades y, finalmente, la aceptación del crédito a la tasa de interés y tiempo establecido entre las partes interesadas. Una vez aprobados los créditos, el Banco del Estado realiza el desembolso periódico de recursos durante el tiempo acordado.

El monto de las aprobaciones en este subsector se ha incrementado drásticamente a partir del año 2006. Si entonces alcanzan 18,8 millones de dólares, en 2007 ascendieron a 68,8 millones y en 2010 estaban en el orden de los 245,5 millones. Seguidamente, se observa un comportamiento a la baja hasta el año 2013, fecha en la que se sitúan en 153,0 millones. En el año 2014 las aprobaciones en agua y saneamiento alcanzaron su punto más alto, registrándose 408,1 millones de dólares. Con el cambio de la política en 2015, las aprobaciones cayeron hasta los 238, 4 millones ( $42 \%$ menos que el año anterior).

TABLA 6.

Aprobaciones Del SeCtor DE AGUa y SANEAMIENTO (2006-20I 5 )

\begin{tabular}{lcccccccccc}
\hline \multicolumn{1}{c}{ Ańo } & $\mathbf{2 0 0 6}$ & $\mathbf{2 0 0 7}$ & $\mathbf{2 0 0 8}$ & $\mathbf{2 0 0 9}$ & $\mathbf{2 0 1 0}$ & $\mathbf{2 0 1 1}$ & $\mathbf{2 0 1 2}$ & $\mathbf{2 0 1 3}$ & $\mathbf{2 0 1 4}$ & $\mathbf{2 0 1 5}$ \\
\hline $\begin{array}{l}\text { Aprobaciones } \\
\text { (millones de } \\
\text { dólares) }\end{array}$ & 18,8 & 68,8 & 127,5 & 50,8 & 245,5 & 222,5 & 193,3 & 153,0 & 408,1 & 238,4 \\
\hline $\begin{array}{l}\text { Participación } \\
\text { sobre el total de } \\
\text { aprobaciones (\%) }\end{array}$ & 7,1 & 56,4 & 21,7 & 16,2 & 36,5 & 33,3 & 42,6 & 25,5 & 50,2 & 38,7 \\
\hline
\end{tabular}

Fuente: elaboración propia con base en Banco del Estado (2016). 


\section{CONCLUSIONES}

Desde los enfoques clásicos, el problema del cambio de la política ha sido concebido como un objeto que puede ser estudiado directamente por el investigador para establecer su causalidad. Esta investigación intenta evitar dicha objetivación, que usualmente distorsiona el objeto de estudio (Zittoun, 2009), a través del análisis de los bordes de las redes de política, de los límites que muestra el consenso adquirido entre los actores. Para ello, hemos puesto a prueba la noción de autoridad política y la potente agencia de un actor capaz de ejercer jerarquía y dominio dentro del proceso de toma de decisiones. Esto implica como novedad adentrarse en la politics de la política pública y no solo quedarse en la superficie de la policy. En este sentido, este trabajo no se limita a la descripción de cómo cambian los instrumentos, sino que intenta explicar el cambio de primer orden de la política pública desde sus causas y consecuencias para afrontar el cambio.

El enfoque de redes de política ha sido más utilizado para explicar la gobernanza horizontal de las políticas públicas (Borzel, 1998; Eberhard et al., 2017; Gadda et al., 2019). Sin embargo, en este texto mostramos la utilidad del enfoque en casos de gobernabilidad que implican la intervención top-down de un actor con autoridad. Así, mostramos por qué la intervención directa de la autoridad política reemplaza las creencias programáticas de los otros actores y con ello moldea «directamente los elementos del diseño de la política cuando el núcleo decisional del subsistema se omite y la toma de decisiones sube a un nivel superior o arena de acción exterior» (Real Dato, 2009: 136). En este caso, la intervención del presidente puede ser explicada por la aplicación del "poder autoritario" al ejercer el control sobre la política y sus actores mediante el uso de relaciones de dominación (Knoke, 1993).

Este caso de estudio demuestra que la figura del presidente de la república define tanto la estabilidad como el cambio de la política. Mientras el presidente apoya el principio constitucional de convertir al agua en un derecho y de planificar el servicio a nivel nacional, la política se estabiliza y permite que más actores e instituciones se sumen a la política pública. Sin embargo, cuando sus ideas programáticas cambian, es capaz de desarticular la red y anular la participación de los actores y de las instituciones. De las siete redes de política que se organizaron en el período analizado, solo quedaron dos después de la intervención autoritaria del presidente. La agencia directa del presidente transforma los procesos de toma de decisiones y la presión de su autoridad política genera una lógica altamente centralizada en un modelo jerárquico de toma de decisiones que no admite ninguna contradicción a la voluntad del líder del poder ejecutivo.

\section{Referencias}

Albornoz, María Belén. 2015. «Las redes de política», en Gustavo Endara (ed.), El rol del Estado: contribuciones al debate. Quito: SENPLADES-FES-ILDIS. 
Baumgartner, Frank y Bryan Jones. 2002. Policy Dynamics. Chicago: University of Chicago Press.

BDE (Banco del Estado). 2013. Presentación del Programa Prosaneamiento en el Enlace Ciudadano 325. Disponible en: https:/www,youtube,com/watch?v=SPhjNSOM$9 \mathrm{gU} \& \mathrm{t}=166 \mathrm{~s}$.

BDE (Banco del Estado). 2014. Verónica Gallardo Enlace Ciudadano 378. Disponible en: https://www,youtube,com/watch?v=EOiBjGoVfn0.

BDE (Banco del Estado). 2016. Estadisticas de desembolsos y aprobaciones.

Borzel, Tanja. 1998. "Organizing Babylon. On the Different Conceptions of Policy Networks», Public Administration 76: 253-273. Disponible en: https://doi. org/10.1111/1467-9299.00100.

Bressers, Hans y Laurence O’Toole. 1998. "The selection of policy instruments: a network-based perspective», Journal of Public Policy, 18 (3): 213-239. Disponible en: https://doi.org/10.1017/S0143814X98000117.

Bressers, Hans y Laurence O’Toole. 2007. «Instrument selection and implementation in a networked context», en Pearl Eliadis, Margaret Hill y Michael Howlett (eds.), Designing Government. Montreal: McGuill-Queen's University Press.

Capano, Gilberto. 2009. "Understanding policy change as an epistemological and theoretical problem", Journal of Comparative Policy Analysis: Research and Practice, 11 (1): 7-31. Disponible en: https://doi.org/10.1080/13876980802648284.

Eberhad, Rachel, Richard Margerum, Vella Karen, Severine Mayere y Bruce Taylor 2017. "The Practice of Water Policy Governance Networks: An International Comparative Case Study", Journal Society and Natural Resources, 30 (4): 453-470. Disponible en: https:/doi.org/10.1080/08941920.2016.1272728.

Emerson, Richard. 1962. «Power-Dependence Relations», American Sociological Review, 27 (1): 31-41. Disponible: https://doi.org/10.2307/2089716.

Fontaine, Guillaume. 2015. «Lecciones de América Latina sobre las dimensiones racionales, cognitivas e institucionales del cambio de políticas", Revista de Ciencias Sociales Iconos, 53: 11-30. Disponible en: https://doi.org/10.17141/iconos. 53.2015.1518.

Gadda, Andressa, Juliet Harris, Kay Tisdall y Elisabeth Millership. 2019. «Making children's rights real: Lessons from policy networks and contribution analysis», The International Journal of Human Rights, 23 (3): 393-407. Disponible en: https://doi.org/10.1080/13642987.2018.1558988.

George, Alexander y Andrew Bennett. 2004. Case studies and theory development in the social sciences. Cambridge: MIT Press.

Gerring, John 2008. "Case selection for case study analysis: qualitative and quantitative techniques», en Janet Box-Steffensmeir, Henry Brady y David Collier (eds.), The Oxford Handbook of political methodology. Oxford: Oxford University Press. Disponible en: https://doi.org/10.1093/oxfordhb/9780199286546.001.0001.

Hall, Peter. 1993. "Policy Paradigms, Social Learning, and the State: The Case of Economic Policymaking in Britain», Comparative Politics, 25 (3): 275-296. Disponible en: https://doi.org/10.2307/422246. 
Howlett, Michael, Jonathan Kim y Paul Weaver. 2006. «Assessing Instrument Mixes through Program and Agency-Level Data: Methodological Issues in Contemporary Implementation Research", Review of Policy Research, 23 (1): 129-151. Disponible en: https://doi.org/10.1111/j.1541-1338.2006.00189.x.

Howlett, Michael, Ishani Mukherjee y Joop Koppenjan. 2017. «Policy learning and policy networks in theory and practice: The role of policy brokers in the Indonesian biodiesel policy network", Policy and Society, 36 (2): 233-250. Disponible en: https://doi.org/10.1080/14494035.2017.1321230.

Kingdon, John. 2003. Agendas, Alternatives, and Public Policies. Nueva York: Longman. Disponible en: https://doi.org/10.1093/oxfordhb/9780199646135.013.18.

Klijn, Erik. 1998. Redes de politicas públicas: una visión general. Londres: Sage.

Klijn, Eric Hans y Joop Koppenjan. 2016. Governance networks in the public sector. Oxon: Routledge. Disponible en: https://doi.org/10.4324/9781315887098.

Kisby, Ben. 2007. "Analysing policy networks, towards an ideational approach", Policy Studies, 28 (1): 71-90. Disponible en: https://doi.org/10.1080/01442870601121502.

Knoke, David. 1993. "Networks as political glue», en William Julios Wilson (ed.), Sociology and the public agenda. Londres: Sage. Disponible en: https://doi. org/10.4135/9781483325484.

Knoke, David y Son Yang. 2008. Social network analysis. Londres: Sage. Disponible en: https://doi.org/10.4135/9781412985864.

Lieberman, Ann. 2000. "Networks as learning communities», Journal of Teacher Education, 51: 221-227. Disponible en: https://doi.org/10.1177/002248710005 1003010.

Marsh, David y Rhodes, Roderick A. W. 1992. Policy networks in British politics. A critique of existing approaches. Oxford: Oxford Scholarship Online. Disponible en: https://doi.org/ 10.1093/acprof:oso/9780198278528.001.0001.

Marsh, David y Martin Smith. 2000. «Understanding policy networks: towards a dialectical approach», Political Studies, 48: 4-21. Disponible en: https://doi. org/10.1111/1467-9248.00247.

Méndez, José. 2010. «La política pública como variable dependiente: hacia un análisis más integral de las políticas públicas», en Luis Aguilar (ed.), Políticas Públicas. Ciudad de México: Siglo XXI.

Montpetit, Éric. 2005. "A policy network explanation of biotechnology policy differences between the United States and Canada», Journal of Public Policy, 25 (3): 339-366. Disponible en: https://doi.org/10.1017/S0143814X05000358.

Ordoñez-Matamoros, Gonzalo. 2013. Manual de análisis y diseño de políticas públicas. Bogotá: Universidad Externado de Colombia.

Rayner, Jeremy. 2009. "Understanding policy change as a historical problem», Journal of Comparative Policy Analysis: Research and Practice, 11 (1): 83-96. Disponible en: https://doi.org/10.1080/13876980802648326.

Real Dato, José. 2009. «Mechanisms of policy change: A proposal for a synthetic explanatory framework", Journal of Comparative Policy Analysis: Research and Practice,11(1):117-143.Disponibleen:https://doi.org/10.1080/13876980802648268. 
Richardson, Jeremy. 2000. "Government, Interest Groups and Policy Change», Political Studies, 48: 1006-1025. Disponible en: https://doi.org/10.1111/14679248.00292.

Sabatier, Pau. 1999. "The Advocacy Coalition Framework: An Assessment», en Paul Sabatier (ed.), Theories of the policy process. Boulder: Westview Point Boulder.

Sanz, Luis. 2003. "Análisis de redes sociales: o cómo representar las estructuras sociales subyacentes", Apuntes de Ciencia y Tecnología. 7.

Secretaría Nacional de Planificación y Desarrollo (SENPLADES). 2014a. Agua potable y alcantarillado para erradicar la pobreza en el Ecuador. Quito: SENPLADES.

Secretaría Nacional de Planificación y Desarrollo (SENPLADES). 2014b. Erradicación de la pobreza: agua y saneamiento. Documento de trabajo no publicado.

Secretaría Nacional de Planificación y Desarrollo (SENPLADES). 2014c. Metodología de cálculo de la nueva meta para agua y alcantarillado. Documento de trabajo no publicado.

Secretaría Nacional de Planificación y Desarrollo (SENPLADES). 2014d. Estrategia Nacional para la Igualdad y la Erradicación de la Pobreza. Quito: El Telégrafo.

Secretaría Nacional del Agua (SENAGUA). 2015. Estrategia Nacional de Agua y Saneamiento. Quito.

Zittoun, Philippe. 2009. "Understanding Policy Change as a Discursive Problem», Journal of Comparative Policy Analysis: Research and Practice, 11(1): 65-82. Disponible en: https://doi.org/10.1080/13876980802648235.

\section{Documentos}

Código Orgánico de Organización Territorial Autonomía y Descentralización. 2011. Constitución de la República del Ecuador. 2008.

Texto Unificado de Legislación Ambiental Secundaria. 2006.

\section{Entrevistas}

- Entrevista con actor clave de la Asociación de Municipalidades del Ecuador (EACA). 2016. Entrevista realizada a María Fernanda Maldonado, exdirectora ejecutiva. Entrevista realizada el 25 de febrero de 2016.

- Entrevista con actor clave de la Secretaría Nacional del Agua. (EACS). 2016. Entrevista realizada a Patricia Aguilar, especialista de Fortalecimiento a la Descentralización de Servicios de Agua Potable y Saneamiento. Entrevista realizada el 18 de marzo de 2016.

Presentado para evaluación: 12 de septiembre de 2018.

Aceptado para publicación: 31 de octubre de 2019. 
W. SANTIAGO ALBUJA-ECHEVERRÍA wsalbuja@gmail.com

Ingeniero en Ciencias Económicas y Financieras y magíster en Políticas Públicas (FLACSO Ecuador). Ha trabajado en la función pública desde hace diez años, donde ha desempeñado varios cargos directivos relacionados con la investigación, seguimiento y evaluación. Sus temas de investigación están relacionados con la economía y las políticas sociales.

\section{MARÍA BELÉN ALBORNOZ}

balbornoz@flacso.edu.ec

Profesora-investigadora de la Facultad Latinoamericana de Ciencias Sociales (FLACSO Ecuador), donde actualmente coordina el doctorado de Políticas Públicas. Se especializa en estudios de ciencia, tecnología y sociedad y en el análisis de políticas públicas. Sus temas de investigación están relacionados con las redes de política, las políticas de ciencia, tecnología e innovación, y el análisis sociotécnico. 